\title{
Linear Pharmacokinetic Equations Allowing Direct Calculation of Many Needed Pharmacokinetic Parameters from the Coefficients and Exponents of Polyexponential Equations Which Have Been Fitted to the Data
}

\author{
John G. Wagner ${ }^{1,2}$ \\ Received Jan. 13, 1976-Final Apr. 27, 1976
}

\begin{abstract}
It is shown that if the numerical values of the coefficients and exponents of the polyexponential equation describing the whole blood (plasma or serum) concentration after administration of a drug by bolus intravenous injection, or during or after termination of a constant-rate intravenous infusion, are known, then many needed pharmacokinetic parameters may be obtained directly. Parameters readily calculated by simple arithmetic are as follows: plasma or serum clearance, $\mathrm{Cl}_{\mathrm{p}}$; volume of plasma compartment, $\mathrm{V}_{\mathrm{p}}$; volume of distribution at steady state, $\mathrm{V}_{\mathrm{d} s \mathrm{~s}}, \mathrm{~V}_{\mathrm{darea}}$ or $\mathrm{V}_{\beta}$, extrapolated volume of distribution, $\mathrm{V}_{\text {dexi }} ;$ half-life of elimination, $\mathrm{t}_{1 / 2} ;$ amount metabolized and/or excreted to time $\mathrm{t},\left(\mathrm{A}_{\mathrm{e}}\right)$; amount in the body at time $\mathrm{t}, \mathrm{A}_{\mathrm{b}}$; amount in the plasma (reference) compartment at time $\mathrm{t}, \mathrm{A}_{\mathrm{p}}$; and amount in other compartments at time $\mathrm{t}, \mathrm{A}_{\mathrm{o}}$. Simulations have shown that the equations yield the correct answers for an $\mathrm{n}$-compartment mammillary model with central compartment elimination only, when rate constants, dose, and a value of $\mathrm{V}_{\mathrm{p}}$ have been assigned. Since whole blood (plasma or serum) concentration-time data always lead to ambiguities as to which specific model is involved, the equations are most appropriate.
\end{abstract}

KEY WORDS: direct calculation of pharmacokinetic parameters; use of fitted polyexponential equations; volumes of distribution; plasma clearance.

\section{INTRODUCTION}

In a linear pharmacokinetic system, plasma concentrations following bolus intravenous injection, $C_{p}^{\text {i.v. }}$, will be described by a polyexponential

Supported in part by Public Health Service Grant 5P11 GM 15559.

${ }^{1}$ College of Pharmacy and Upjohn Center for Clinical Pharmacology, The University of Michigan, Ann Arbor, Michigan 48109.

${ }^{2}$ Address reprint requests to Dr. John G. Wagner, Upjohn Center for Clinical Pharmacology, University of Michigan Medical Center, Ann Arbor, Michigan 48109. 
equation as in equation 1. Expanded forms of equation 1, corresponding to one, two, and three exponential terms are shown as equations $2-4$, respectively.

$$
\begin{aligned}
& C_{p}^{\text {i.v. }}=\sum C_{i} e^{-\lambda_{i} t} \\
& C_{p}^{\text {i.v. }}=C_{1} e^{-\lambda_{1} t} \\
& C_{p}^{\text {i.v. }}=C_{1} e^{-\lambda_{1} t}+C_{2} e^{-\lambda_{2} t} \\
& C_{p}^{\text {i.v. }}=C_{1} e^{-\lambda_{1} t}+C_{2} e^{-\lambda_{2} t}+C_{3} e^{-\lambda_{3} t}
\end{aligned}
$$

In equations $1-4, C_{i}\left(C_{1}, C_{2}, C_{3}\right)$ are the coefficients, $\lambda_{i}\left(\lambda_{1}, \lambda_{2}, \lambda_{3}\right)$ are the exponents, and $t$ is time.

The numerical values of the $C_{i}$ 's and $\lambda_{i}$ 's of equation 1 obtained from a given set of data depend on (a) the specific linear pharmacokinetic model (including exactly how many exit rate constants there are and where they exit from the one or more compartments), (b) the numerical values of the rate constants ( $k_{i j}$ 's and $k_{i o}$ 's), (c) the dose, $D$, of the drug, and (d) the volume of the plasma (reference) compartment, $V_{p}$.

In another article (1), it is shown that one and sometimes two terms of the polyexponential equation describing the plasma concentration after either intravenous or oral administration can readily vanish a few minutes after administration. This fact, coupled with the more complicated, nonclassical linear models discussed in that article, indicates that most of the time we cannot even determine which class of model we are dealing with. Also, there is no a priori reason to assume that all subjects or patients in a panel should have the same model for a drug.

When only whole blood (plasma or serum) concentration-time data and/or urinary excretion data are available, there is really no rigorous method to determine whether elimination occurs only from the central (reference) compartment or from one or more of the peripheral compartments or both. Thus when evaluating such data the pharmacokineticist is presented with a dilemma. We have really not confronted this problem adequately to date. It has become customary in pharmacokinetics to assume one specific model for a given drug administered to a panel of subjects or patients, then to "force this specific model" on all sets of data, and to derive various kinetic parameters. There are three two-compartment open models, all of which give an equation of the form of equation 3 after bolus intravenous injection. There are 21 three-compartment open models, all of which give an equation of the form of equation 4 after bolus intravenous injection. We do not have the methods available to determine from the $C_{i}$ 's and $\lambda_{i}$ 's of equation 1 which class of models or which specific model applies to a given set of data in either of these situations. In fact, relative to the 
disposition model, unless special sampling procedures are used to detect peripheral metabolism or excretion, we have no recourse but to assume that all elimination processes occur only from the central compartment.

Three proposals are being made in this article. These are as follows:

1. Assume (unless there is strong evidence to the contrary) that the general model which applies is an $n$-compartment mammillary model with elimination only from the central (reference) compartment as shown in Scheme I. The methods given for calculation of all pharmacokinetic parameters in this article apply to this general model. (See the discussion section for further details.)

2. Obtain a nonlinear least-squares fit of each set of data to the general equation 1, but use the number of terms required for each set. Don't "force" all data sets in a group to either a biexponential or a triexponential equation. Reevaluation of many sets of literature data has revealed that data from some members of a panel require only one exponential term, others require two exponential terms, and others require three exponential terms; such results will be reported in a subsequent article.

3. Calculate values of the pharmacokinetic parameters, such as $V_{p}$, $V_{d \mathrm{ss}}, V_{d \mathrm{area}}, V_{d \mathrm{ext}}, \mathrm{Cl}_{p}$, and $t_{1 / 2}$ (see next section) directly from the coefficients and exponents of the fitted polyexponential equation. It is best to express the dose in $\mathrm{mg} / \mathrm{kg}$ if the concentration data are in

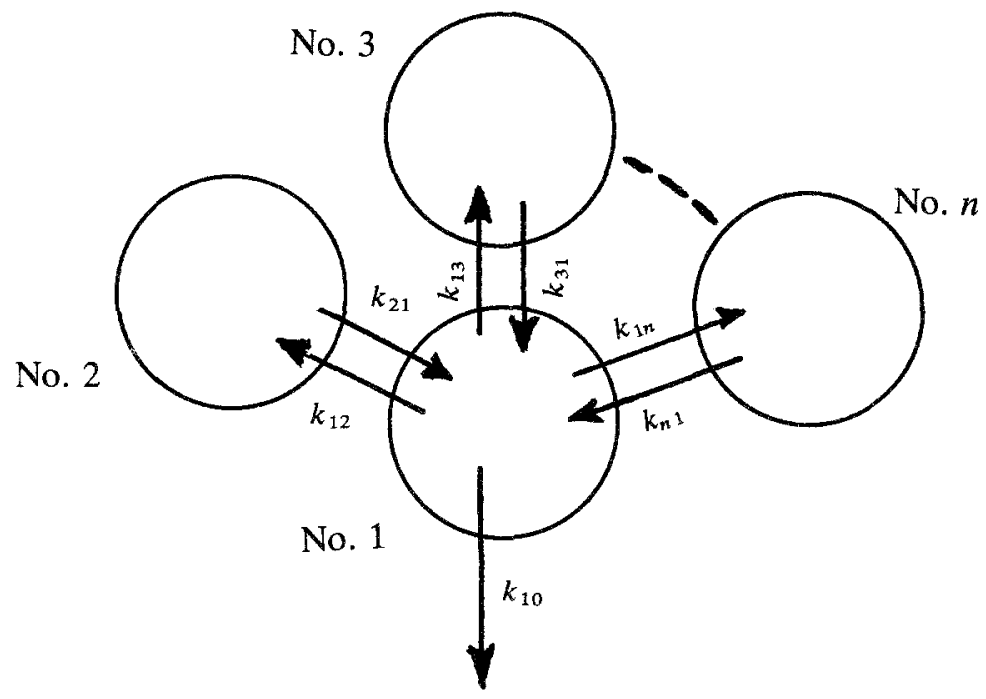

Scheme I. The $n$-compartment open mammillary model on which the equations are based. 
$\mu \mathrm{g} / \mathrm{ml}$, and in $\mu \mathrm{g} / \mathrm{kg}$ if the concentration data are in $\mathrm{ng} / \mathrm{ml}$, so that the volumes will have dimensions of liters $/ \mathrm{kg}$; such values usually have smaller coefficients of variation than values in liters obtained from the same data.

\section{THEORETICAL}

\section{Symbolism}

$A_{b}$ is the amount of drug in the "body" (i.e., all compartments) at time $t$. $\left(A_{b}\right)_{\beta}$ is the amount of drug in the "body" (all compartments) at time $t$ in the terminal log-linear phase (i.e., only $C_{1} e^{-\lambda_{1} t}$ is still contributing).

$A_{b}^{\mathrm{ss}}$ is the amount of drug in the "body" (all compartments) at time $t$ at steady state.

$\widehat{A_{b}^{\text {ss }}}$ is the average amount of drug in the "body" (all compartments) at steady state.

$A_{e}$ is the amount of drug which has been eliminated (metabolized and/or excreted) from the "body" in time $t$.

$A_{p}$ is the amount of drug in the plasma (reference) compartment at time $t$.

$A_{o}=A_{b}-A_{p}$ is the amount of drug in compartments other than the plasma or reference compartment at time $t$.

AUC is the area under the plasma concentration-time curve (subscripts give limits).

$B_{i}(i=1,2, \ldots, n)$ is the coefficient of the $i$ th exponential term of the polyexponential equation describing the plasma concentration following oral administration (i.e., analogous to equation 1 except that $B_{i}$ replaces $\left.C_{i}\right)$.

$C_{i}(i=1,2, \ldots, n)$ is the coefficient of the $i$ th exponential term of the polyexponential equation describing the plasma concentration following bolus intravenous administration.

$\mathrm{Cl}_{p}$ is the plasma clearance (dimension of volume/time).

$\mathrm{C}_{p}$ is the plasma concentration (i.e., concentration in the reference compartment with volume $V_{p}$ ) at time $t$. $C^{\text {i.v. }}$ refers to bolus intravenous administration and $C_{p}^{\text {p.o. }}$. refers to oral administration.

$C_{p}^{\mathrm{ss}}$ is the plasma concentration at time $t$ at steady state.

$\overline{C_{p}^{s s}}$ is the average plasma concentration at steady state.

$D$ is the dose of drug administered. For a constant-rate infusion $D=k_{0} T . D_{\text {p.o. }}$ refers to oral administration and $D_{\text {i.v. }}$ refers to bolus intravenous administration. 
$D_{L}$ is the loading dose.

$D_{M}$ is the maintenance dose.

$E_{i j}$ is the exit rate constant for the $i$ th compartment and equals the sum of all first-order rate constants whose arrowheads point away from the $i$ th compartment.

$F$ is the bioavailability factor concerned with incomplete absorption of the dose.

$F^{*}$ is the bioavailability factor concerned with the so-called first-pass effect. This is defined by equation 28 when $F=1$. For a given model, $F^{*}$ is a combination of rate constants and $F^{*} \leq 1$.

$f$ is the fraction of the drug which is free in the whole body.

$k_{i j}$ is a first-order "microscopic rate constant" for transfer of drug from the $i$ th to the $j$ th compartment.

$k_{i o}$ is a first-order "microscopic rate constant" for transfer of drug from the $i$ th compartment to outside "the body" or elimination rate constant of the $i$ th compartment.

$k_{0}$ is the constant intravenous infusion rate (dimensions of mass/time).

$\lambda_{i}$ is the exponent multiplying $t$ in the exponential terms. Usually each $\lambda_{i}$ will be an eigenvalue which is the absolute value of one of the roots when the determinant is set equal to zero; in this case, the $\lambda_{i}$ is a function of all or many of the $k_{i j}$ 's and $k_{i o}$ 's of the model. In some cases, the $\lambda_{i}$ is equal to one of the $k_{i j}$ 's. Note that $\lambda_{1}$ is the smallest $\lambda_{i}$ (classically symbolized by $\beta$ ), $\lambda_{2}$ is the second smallest $\lambda_{i}$, etc.; i.e., the order of $\lambda_{i}$ 's with respect to magnitude is the same as that obtained by stripping the data. Note that $\lambda_{i}$ refers to bolus intravenous administration or constant infusion and $\lambda_{i}^{\text {p.o. }}$ refers to oral administration.

$Q_{i}(i=1$ or 2$)$ are infusion rates (see equations 31 and 32 and text nearby).

$S_{i}(i=1, \ldots, 5)$ are particular sums defined by equations $5-9$. $\sum=\sum_{i=1}^{n}$.

$\sigma$ is the fraction of the drug which is free (not protein bound) in plasma.

$t_{1 / 2}$ is the apparent elimination half-life of the drug.

$T$ is the duration of a constant-rate intravenous infusion (dimension of time).

$\tau$ is the uniform dosage interval (dimension of time).

$t_{0}$ is a lag time sometimes introduced into a polyexponential equation for $C_{p}^{\text {p.o. }}$ for oral administration (dimensions of time).

$t_{\mathrm{ss}}^{\mathrm{max}}$ is the time of the maximum plasma concentration after oral dosing at steady state.

$V_{\beta}$ or $V_{d \text { area }}$ is defined by equations 6 and 13 . 
$V_{d s \mathrm{~s}}$ is the volume of distribution at steady state (based on the model of Scheme I).

$V_{d \mathrm{ss}} C_{p}^{\mathrm{ss}}$ is the amount of drug in the body at time $t$ at steady state.

$V_{d s s} C_{p}^{s s}$ is the average amount of drug in the body at steady state.

$V_{p}$ is the volume of the plasma (reference) compartment.

$X_{i}$ is the coefficient of the $i$ th exponential term of the polyexponential equation giving the plasma concentration during constant-rate intravenous infusion.

$Y_{i}$ is the coefficient of the $i$ th exponential term of the polyexponential equation giving the plasma concentration after a constant rate infusion has ceased.

\section{Equations}

Equations for Bolus Intravenous Injection ${ }^{3}$

The plasma concentration is described by equation 1 .

Let

$$
\begin{aligned}
& S_{1}=\sum C_{i} \\
& S_{2}=\sum C_{i} / \lambda_{i} \\
& S_{3}=\sum C_{i} / \lambda_{i}^{2} \\
& S_{4}=\sum \frac{C_{i}}{\lambda_{i}} e^{-\lambda_{i} t} \\
& S_{5}=\sum \frac{C_{i}}{\lambda_{i}}\left(1-e^{-\lambda_{i} t}\right)
\end{aligned}
$$

Then the plasma clearance, $\mathrm{Cl}_{p}$, is defined by equation 10 .

$$
\mathrm{Cl}_{p}=D_{\text {i.v. }} / S_{2}=D_{\text {i.v. }} / \mathrm{AUC}_{0 \rightarrow \infty}
$$

The volume of the plasma compartment, $V_{p}$, is given by equation 11 .

$$
V_{p}=D_{\text {i.v. }} / S_{1}
$$

The volume of distribution at steady state, $V_{d \mathrm{~s} s}$, is given by equation 12 .

$$
V_{d \mathrm{ss}}=D_{\text {i.v. }} S_{3} /\left(S_{2}\right)^{2}
$$

$V_{d \text { area }}$ or $V_{\beta}$ is given by equation 13 .

$$
V_{\text {darea }}=D_{\text {i.v. }} / \lambda_{1} S_{2}=D / \lambda_{1}\left(\mathrm{AUC}_{0 \rightarrow \infty}\right)
$$

${ }^{3}$ See Appendix for some derivations. 
The extrapolated volume of distribution, $V_{\text {dext }}$, is given by equation 14 .

$$
V_{d \mathrm{ext}}=D / C_{1}
$$

The elimination half-life, $t_{1 / 2}$, is given by equation 15 .

$$
t_{1 / 2}=0.693 / \lambda_{1}
$$

The amount which has been metabolized and/or excreted to time $t, A_{e}$, is given by equation 16 .

$$
A_{e}=D_{\text {i.v. }} S_{5} / S_{2}=D\left(\mathrm{AUC}_{0 \rightarrow t}\right) /\left(\mathrm{AUC}_{0 \rightarrow \infty}\right)
$$

It should be emphasized that equation 16 is mathematically correct only when elimination occurs solely from the central compartment. The amount of drug in the body at time $t, A_{b}$, is given by equation 17 (1).

$$
A_{b}=D_{\text {i.v. }} S_{4} / S_{2}=\left(V_{d}\right)_{t} \cdot C_{p}=D\left(\mathrm{AUC}_{t \rightarrow \infty}\right) /\left(\mathrm{AUC}_{0 \rightarrow \infty}\right)
$$

The amount of drug in the plasma compartment at time $t, A_{p}$, is given by equation 18.

$$
A_{p}=D_{\text {i.v. }} C_{p}^{\text {i.v. }} / S_{1}=V_{p} C_{p}^{\text {i.v. }}
$$

The amount of drug in other compartments than the plasma compartment at time $t, A_{o}$, is given by equation 19 .

$$
A_{o}=A_{b}-A_{p}
$$

Equations 19 , in effect, lumps $n$ peripheral compartments into one peripheral compartment.

The time-dependent volume of distribution, $\left(V_{d}\right)_{t}$, is given by equation 20 , originally derived by Niazi (2).

$$
\left(V_{d}\right)_{t}=A_{b} / C_{p}=D_{\mathrm{i} . v .} S_{4} / C_{p}^{\mathrm{i} . v} \cdot S_{2}=D\left(\mathrm{AUC}_{t \rightarrow \infty}\right) / C_{p}\left(\mathrm{AUC}_{0 \rightarrow \infty}\right)
$$

It should be noted that the order of magnitudes of the volumes is always

$$
V_{d \mathrm{ext}}>V_{d \mathrm{area}}>V_{d \mathrm{ss}}>V_{p}
$$

It should also be noted that $\left(A_{b}\right)_{\beta}=V_{d \text { area }} \cdot C_{p}$, since $\left(V_{d}\right)_{t}=V_{d \text { area }}$ in the log-linear phase when all exponential terms, except $e^{-\lambda_{1} t}$, essentially equal zero.

\section{Relationship Between Bolus Intravenous and Infusion Equations}

The relationships between the bolus intravenous and intravenous infusion equations are shown in Table $I$ for expansions up to and including three exponential terms. During a constant-rate intravenous infusion $(t<T)$ the 
plasma concentration is described by equation 21 , and after the infusion has ceased $(t>T)$ the plasma concentration is described by equation 22 .

$$
\begin{aligned}
& C_{p}=\sum X_{i}\left(1-e^{-\lambda_{i}}\right)=\sum \frac{C_{i}}{\lambda_{i} T}\left(1-e^{-\lambda_{i} t}\right) \\
& C_{p}=\sum Y_{i} e^{-\lambda_{i} t}=\sum C_{i}\left\{\frac{e^{+\lambda_{i} T}-1}{\lambda_{i} T}\right\} e^{-\lambda_{i} t}
\end{aligned}
$$

By matching coefficients, we see that

$$
\begin{gathered}
X_{i}=C_{i} / \lambda_{i} T \\
Y_{i}=C_{i}\left\{\frac{e^{+\lambda_{i} T}-1}{\lambda_{i} T}\right\}
\end{gathered}
$$

From equation 23 we see that $C_{i}=\lambda_{i} T X_{i}$, and from equation 24 we obtain equation 25 .

$$
C_{i}=\frac{\lambda_{i} T Y_{i}}{e^{+\lambda_{i} T}-1}
$$

Thus, if "during infusion" data are fitted to the $X_{i}$ form of equation 21 and/or "postinfusion" data are fitted to the $Y_{i}$ form of equation 22, one can readily obtain the coefficients, $C_{i}$, corresponding to bolus intravenous injection and then apply equations 5-20 to obtain the pharmacokinetic parameters. Corrections are needed with equation 25 even when infusions as short as $5 \mathrm{~min}$ are given and postinfusion data are evaluated.

\section{Bioavailability}

Equation 26 symbolizes the result obtained by fitting a set of $C_{p}^{\text {p.o. }}, t$ data obtained following oral administration, if the system is linear.

$$
C_{p}^{\text {p.o. }}=\sum B_{i} e^{-\lambda \text { p.o. }\left(t-t_{0}\right)}=\sum B_{i}^{\prime} e^{-\lambda \stackrel{p}{p} \cdot \text { o.t }}
$$

where

$$
B_{i}^{\prime}=B_{i} e^{+\lambda \text { p.o. }_{0}}
$$

Equations 26 and 27 include a lag time, $t_{0}$, which may be a positive value or zero. The program CSTRIP(3) automatically determines whether a lag time is needed to describe a given set of oral data. If $t_{0}=0$, then $B_{i}=B_{i}^{\prime}$.

It should be noted that $B_{i}^{\prime}$ can replace $C_{i}$ and $\lambda_{i}^{\text {p.o. }}$ can replace $\lambda_{i}$ in equations 5 and 6 . The result would give the sums $S_{1}^{\text {p.o. }}$ and $S_{2}^{\text {p.o. }}$, respectively. If the latter values are then used in equations 10 and 13 , with substitution of $D_{\text {p.o. }}$ for $D_{\text {i.v. }}$, then $\mathrm{Cl}_{p} / F F^{*}$ and $V_{d \text { area }} / F F^{*}$, respectively, are calculated and not $\mathrm{Cl}_{p}$ and $V_{d \text { area }}$, respectively. It is very common in the 


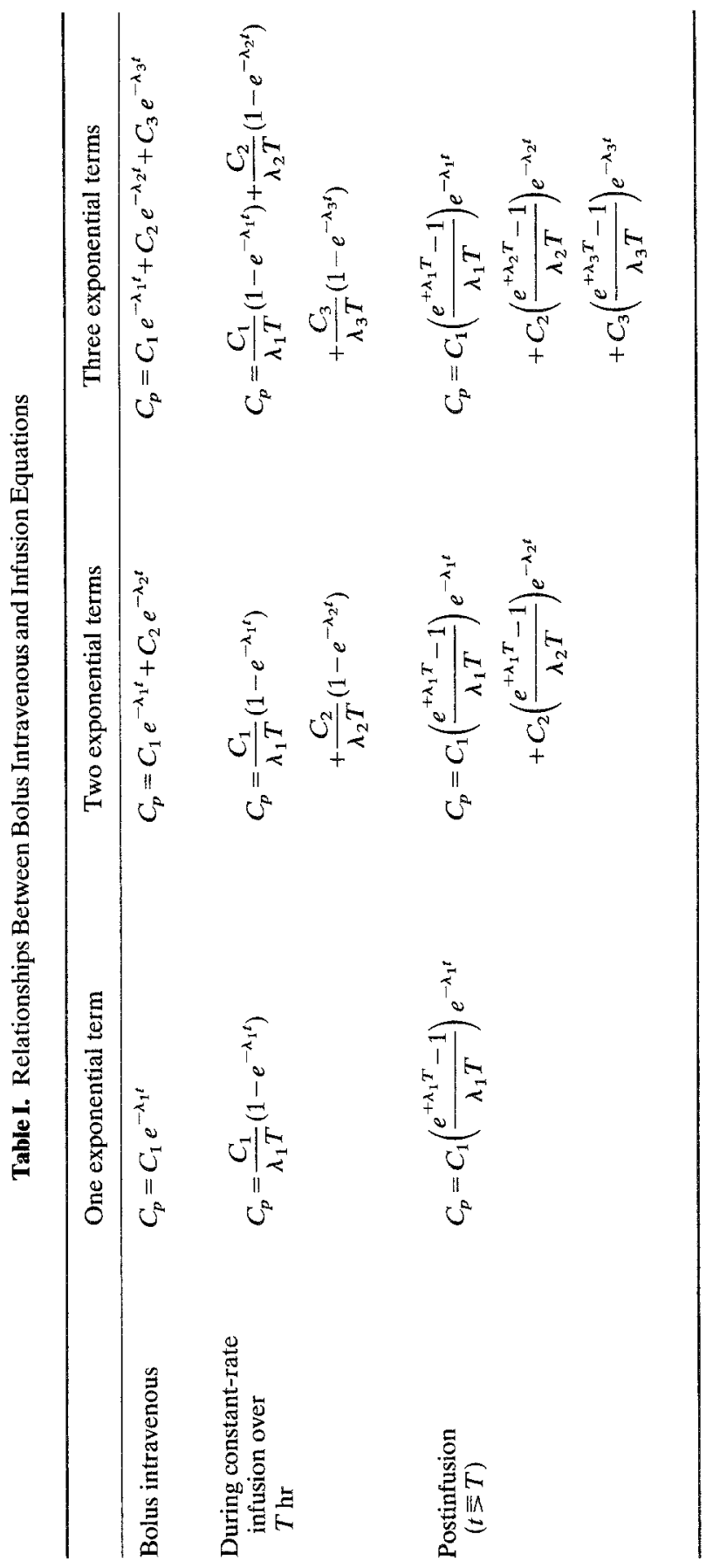


literature to ignore $F^{*}$ completely and assume $F=1$ for oral administration, then claim that the parameters estimated are $\mathrm{Cl}_{p}$ and $V_{d \text { area }}$. This technique just causes confusion.

By definition, the product $F F^{*}$ is given by equation 28 .

$$
F F^{*}=\frac{D_{\text {i.v. }} \int_{0}^{\infty} C_{p}^{\text {p.o. }} d t}{D_{\text {p.o. }} \int_{0}^{\infty} C_{p}^{\text {i.v. }} d t}=\frac{D_{\text {i.v. }} \sum B_{i}^{\prime} / \lambda_{i}^{\text {p.o. }}}{D_{\text {p.o. }} \sum C_{i} / \lambda_{i}}
$$

Thus $F^{*}$ is given by equation 28 when there is complete absorption of the oral dose $(F=1)$.

The value of $F^{*}$ for a given model is obtained by substituting the appropriate expressions for $C_{p}^{\text {i.v. }}$ and $C_{p}^{\text {p.o. }}$ into equation 28 , letting $D_{\text {i.v. }}=$ $D_{\text {p.o. }}$ and $F=1$, then performing the integrations and simplifying the resultant ratio, or by use of Laplace transforms to obtain expressions for the areas.

When real data sets have been fitted by the method of least squares and the numerical values of $D_{\text {i.v. }}, D_{\text {p.o., }}, B_{i}^{\prime}, \lambda_{i}$ are known, they may be substituted into the right-hand side of equation 28 and the value of $F F^{*}$ calculated directly.

\section{Drugs Bound to Plasma Protein and Tissues}

If $\sigma$ is the fraction of the drug which is free (not protein bound) in plasma, $f$ is the fraction of the total drug in the body which is free, $\left(\lambda_{1}\right)_{F}$ is the intrinsic apparent elimination rate constant referenced to free drug, $\lambda_{1}$ is the apparent elimination rate constant obtained from $C_{p}, t$ data, and the onecompartment open model with linear plasma protein binding and linear tissue binding applies, then

$$
\begin{gathered}
\lambda_{1}=f\left(\lambda_{1}\right)_{F} \\
\mathrm{Cl}_{p}=V_{d \mathrm{ext}} \cdot \lambda_{1}=V_{d \mathrm{ext}} \cdot \sigma \cdot\left(\lambda_{1}\right)_{F}
\end{gathered}
$$

For this particular one-compartment open model, $V_{d \text { area }}=V_{d \text { ext }}$ and both depend on both $f$ and $\sigma$. This will be explained in a future publication by the author.

\section{Safe and Rapid Attainment of Steady-State Plasma Concentrations}

Once $\lambda_{1}$ and $\mathrm{Cl}_{p}$ are known for a linear system and a particular subject (or average parameter values are used), then steady-state plasma levels may be safely and rapidly attained by the method of Wagner (4). The steps are as follows:

1. Choose the desired steady-state plasma concentration, $C_{p}^{\text {ss }}$.

2. Choose the time $T$ for the duration of the initial constant-rate intravenous infusion at the rate $Q_{1}$ (mass/time). 
3. Calculate the final infusion rate $Q_{2}$ (mass/time) for $t>T$ with equation 31 .

$$
Q_{2}=\mathrm{Cl}_{p} \cdot C_{p}^{s s}
$$

4. Calculate the initial infusion rate, $Q_{1}$, for $t<T$ with equation 32 .

$$
Q_{1}=Q_{2} /\left(1-e^{-\lambda_{1} T}\right)
$$

Then, at zero time initiate the infusion at the rate $Q_{1}$. At time $T$, abruptly change the rate from $Q_{1}$ to $Q_{2}$. Steady state is reached in about an hour or less after the rate $Q_{2}$ is started. The amount of drug in the body at steady state, $A_{b}^{\mathrm{ss}}$, is given by equation 33 .

$$
A_{b}^{\mathrm{ss}}=V_{d \mathrm{ss}} \cdot C_{p}^{\mathrm{ss}}
$$

\section{Equations for Steady State After Oral Administration (5)}

When steady-state equation corresponding to equation 26 is equation 34 when equal doses are given at equal time intervals, $\tau$. In equation $34, t$ is the time after a dose given at steady state.

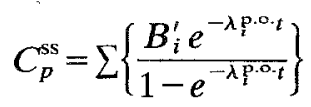

To find the time of the maximum steady-state plasma concentration, $t_{\mathrm{ss}}^{\max }$, one takes the derivative of equation 34 and sets it equal to zero, then solves the equation by use of logarithms if these are only one or two exponential terms, or, iteratively, if there are more than two exponential terms. This is indicated by equation 35 .

$$
\frac{d C_{p}^{\text {ss }}}{d t}=\sum \frac{-\lambda_{i}^{\text {p.o. }} \cdot B_{i}^{\prime} e^{-\lambda p_{i} .0 . t_{\mathrm{ss}}}}{1-e^{-\lambda p_{i}^{0.0 . \tau}}}=0
$$

Once $t_{\mathrm{ss}}^{\max }$ is known, then it may be substituted for $t$ in equation 34 to yield the maximum plasma concentration at steady state, $C_{\mathrm{ss}}^{\max }$, indicated by equation 36 .

$$
C_{\mathrm{ss}}^{\max }=\sum\left\{\frac{B_{i}^{\prime} e^{-\lambda \text { p.o. } \operatorname{tmax}_{\mathrm{ss}}}}{1-e^{-\lambda p_{i} .0 . \tau}}\right\}
$$

The minimum plasma concentration at steady state, $C_{\mathrm{ss}}^{\min }$, is obtained by substituting $\tau$ for $t$ in equation 34, with the result shown as equation 37 .

$$
C_{\mathrm{ss}}^{\min }=\sum\left\{\frac{B_{i}^{\prime} e^{-\lambda p_{i} .0 . \tau}}{1-e^{-\lambda p_{i} .0 . \tau}}\right\}
$$


To attain steady state quickly on oral dosing, the ratio of the loading dose, $D_{L}$, to the maintenance dose, $D_{m}$, is given by equation $38(5)$.

$$
\frac{D_{L}}{D_{m}}=\frac{\int_{0}^{\tau} C_{p}^{\text {ss }} d t}{\int_{0}^{\tau} C_{p} d t}=\sum B_{i}^{\prime} / \lambda_{i}^{\text {p.o. }} / \sum\left[\frac{B_{i}^{\prime}\left(1-e^{-\lambda \text { p.o. })}\right)}{\lambda_{i}^{\text {p.o. }}}\right]
$$

Similar equations to 34-38 may be used for bolus intravenous injections given at intervals of $\tau \mathrm{hr}$, but $B_{i}$ is replaced by $C_{i}$ and $\lambda_{i}^{\text {p.o. }}$ is replaced by $\lambda_{i}$ (i.e., one uses equation 1 in place of equation 26). When the intravenous route is used, then the average steady-state plasma concentration, $\overline{C_{p}^{s s}}$, is given by equation $39(6)$.

$$
\overline{C_{p}^{s s}}=D_{m} /\left(\mathrm{Cl}_{p} \cdot \tau\right)
$$

If one substitutes the maintenance dose, $D_{m}$, the clearance, $\mathrm{Cl}_{p}$, and the dosage interval, $\tau$, into the right-hand side of equation 39 for oral administration, the quantity estimated is $\overline{C_{p}^{s s}} / F F^{*}$ (since drug reaching the circulation is $F F^{*} D_{m}$ and not $D_{m}$ ). However, if one had estimated an apparent plasma clearance, $\mathrm{Cl}_{p} / F F^{*}$, from oral data and used it in place of $\mathrm{Cl}_{p}$ in equation 39 , then $C_{p}^{\frac{5}{s s}}$ would be estimated. There is much confusion in the literature about such calculations.

There is also some confusion in the literature as to what "clearances" mean. Various "clearances" were calculated for diphenhydramine from plasma concentrations measured both during and after a constant-rate intravenous infusion and after oral administration of similar (but known) doses of the drug given as an aqueous solution and in capsule form (7). Results obtained may be summarized as shown in Table II. It is obvious that $F$ and $F^{*}$ are confounded. We can obtain an estimate of $F^{*}$ only if we assume $F_{s}=1$ for the solution given orally.

\section{EXPERIMENTAL}

\section{Example of Obtaining Coefficients of Bolus Intravenous Equations from Postinfusion Data}

For the two-compartment open model with elimination only from the central compartment, let $k_{12}=3, k_{21}=2, k_{\mathrm{el}}=0.1, D_{\mathrm{i} . \mathrm{v} .}=500,000, V_{p}=$ $5000, k_{0}=250,000$, and $T=2$. For these constants, we find $\lambda_{1}=0.03952$ and $\lambda_{2}=5.0605$ (see Appendix). For bolus intravenous injection,

$$
C_{p}=\frac{D_{\text {i.v. }}}{V_{p}\left(\lambda_{2}-\lambda_{1}\right)}\left[\left(k_{21}-\lambda_{1}\right) e^{-\lambda_{1} t}-\left(k_{21}-\lambda_{2}\right) e^{-\lambda_{2} t}\right]
$$




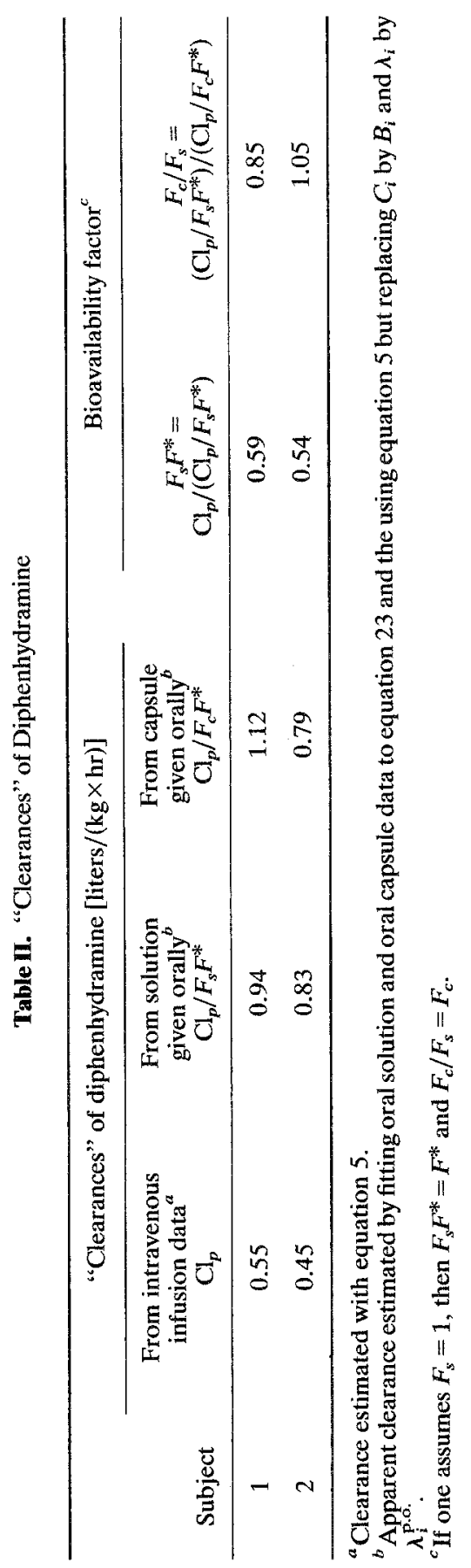


Substitution of the assigned values into equation 40 yields equation 41 .

$$
C_{p}=39.0459 e^{-0.03952 t}+60.9545 e^{-5.0605 t}
$$

Hence $C_{\mathrm{i}}=39.0459$ and $C_{2}=60.9454$.

For postinfusion,

$$
C_{p}=\frac{k_{0}\left(k_{21}-\lambda_{1}\right)\left(1-e^{+\lambda_{1} T}\right)}{-\lambda_{1}\left(\lambda_{2}-\lambda_{1}\right) V_{p}} e^{-\lambda_{1} t}+\frac{k_{0}\left(k_{21}-\lambda_{2}\right)\left(1-e^{+\lambda_{2} T}\right)}{-\lambda_{2}\left(\lambda_{1}-\lambda_{2}\right) V_{p}} e^{-\lambda_{2} t}
$$

Substitution of the assigned values into equation 42 yields equation 43 .

$$
C_{p}=40.6303 e^{-0.03952 t}+149,712.2288 e^{-5.0605 t}
$$

Hence

$$
Y_{1}=40.6303 \quad \text { and } \quad Y_{2}=149,712.2288
$$

Thus

$$
C_{1}=\frac{T \lambda_{1} Y_{1}}{e^{+\lambda_{1} T}-1}=\frac{(2)(0.03952)(40.6303)}{e^{+(0.03952)(2)}-1}=39.0459
$$

and

$$
C_{2}=\frac{T \lambda_{2} Y_{2}}{e^{+\lambda_{2} T}-1}=\frac{(2)(5.0605)(149,712.2288)}{e^{+(5.0605)(2)}-1}=60.9542
$$

The $C_{1}$ and $C_{2}$ values obtained with equations 44 and 45 , respectively, are the same as those in the intravenous equation 41 within round-off error.

\section{Examples Showing That Equation 12 Yields the Correct Value of $\boldsymbol{V}_{d s s}$}

\section{Example 1}

Example 1 is of a two-compartment open model with elimination only from the central compartment.

Let the constants be the same as in the above example, hence equation 41 holds.

Substituting from equation 41 into equations 6,7 , and 12 gives equation 46.

$$
\begin{aligned}
V_{d \mathrm{ss}} & =500,000\left[\frac{39.0459}{(0.03952)^{2}}+\frac{60.9545}{(5.0605)^{2}}\right] /\left[\frac{39.0459}{0.03952}+\frac{60.9545}{5.0605}\right]^{2} \\
& =12,501
\end{aligned}
$$


For this model, $V_{d \mathrm{ss}}$ is known to be given by equation 47 .

$$
V_{d \mathrm{ss}}=\left(1+k_{12} / k_{21}\right) V_{p}=(1+3 / 2) 5000=12,500
$$

The difference of 1 in 12,500 is due only to round-off error in the $C_{i}$ 's and $\lambda_{1}$ 's.

\section{Example 2}

Example 2 is of a three-compartment open model with elimination only from the central compartment shown in Scheme II. The dose is put into compartment 1 at time zero.

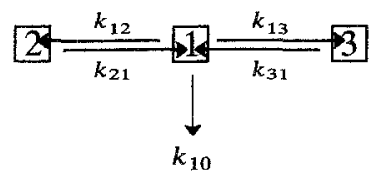

Scheme II

By writing the differential equations for Scheme I, converting to Laplace transforms, and setting the determinant, $\Delta$, equal to zero, one obtains equations $48-51$,

$\Delta=\left|\begin{array}{ccc}\left(S+E_{1}\right) & -k_{21} & -k_{31} \\ -k_{12} & \left(S+k_{21}\right) & 0 \\ -k_{13} & 0 & \left(S+k_{31}\right)\end{array}\right|=S^{3}+a_{2} S^{2}+a_{1} S+a_{0}=0$

where

$$
\begin{aligned}
& a_{2}=E_{1}+k_{21}+k_{31} \\
& a_{1}=E_{1} k_{21}+E_{1} k_{31}+k_{21} k_{31}-k_{12} k_{21}-k_{13} k_{31} \\
& a_{0}=E_{1} k_{21} k_{31}-k_{12} k_{21} k_{31}-k_{13} k_{21} k_{31}
\end{aligned}
$$

There are three negative roots (eigenvalues) of equations $48-51$ and the absolute values of these roots are designated $\lambda_{1}, \lambda_{2}$, and $\lambda_{3}$. The relationships are

$$
\begin{aligned}
& a_{2}=\lambda_{1}+\lambda_{2}+\lambda_{3} \\
& a_{1}=\lambda_{1} \lambda_{2}+\lambda_{1} \lambda_{3}+\lambda_{2} \lambda_{3} \\
& a_{0}=\lambda_{1} \lambda_{2} \lambda_{3}
\end{aligned}
$$


The Laplace transform of the amount of drug in compartment 1 at $t, a_{1}$, is given by equation 55 .

$$
a_{1}=\frac{\left|\begin{array}{ccc}
D & -k_{21} & -k_{21} \\
0 & \left(S+k_{21}\right) & 0 \\
0 & 0 & \left(S+k_{31}\right)
\end{array}\right|}{\Delta}=\frac{D\left(S+k_{21}\right)\left(S+k_{31}\right)}{\left(S+\lambda_{1}\right)\left(S+\dot{\lambda}_{2}\right)\left(S+\lambda_{3}\right)}
$$

Letting $C_{p}^{\text {i.v. }}=A_{1} / V_{p}$, and taking the antitransform of equation 55 , gives

$$
\begin{aligned}
C_{p}^{\text {i.v. }}= & \frac{D_{\text {i.v. }}}{V_{p}}\left[\frac{\left(k_{21}-\lambda_{1}\right)\left(k_{31}-\lambda_{1}\right)}{\left(\lambda_{2}-\lambda_{1}\right)\left(\lambda_{3}-\lambda_{1}\right)} e^{-\lambda_{1} t}+\frac{\left(k_{21}-\lambda_{2}\right)\left(k_{31}-\lambda_{3}\right)}{\left(\lambda_{1}-\lambda_{2}\right)\left(\lambda_{3}-\lambda_{2}\right)} e^{-\lambda_{2} t}\right. \\
& \left.+\frac{\left(k_{21}-\lambda_{3}\right)\left(k_{31}-\lambda_{3}\right)}{\left(\lambda_{1}-\lambda_{3}\right)\left(\lambda_{2}-\lambda_{3}\right)} e^{-\lambda_{3} t}\right]
\end{aligned}
$$

For example, let $k_{12}=3, k_{21}=2, k_{13}=1, k_{31}=0.5, k_{10}=0.1, D_{\text {i.v. }}=$ 500,000 , and $V_{p}=5000$. Then

$$
\begin{aligned}
& E_{1}=0.1+3+1=4.1 \\
& a_{2}=4.1+2+0.5=6.6 \\
& a_{1}=(4.1)(2)+(4.1)(0.5)+(2)(0.5)-(3)(2)-(1)(0.5)=4.75 \\
& a_{0}=(4.1)(2)(0.5)-(3)(2)(0.5)-(1)(2)(0.5)=0.10
\end{aligned}
$$

Substituting from equations 58-60 into equation 48 gives

$$
S^{3}+6.6 S^{2}+4.75 S+0.10=0
$$

An electronic calculator cube root program gave the roots of equation 61 as $-0.021705074,-0.796904816$, and -5.7813901 .

Hence $\lambda_{1}=0.021705074, \lambda_{2}=0.796904816$, and $\lambda_{3}=5.7813901$.

Substituting these values into equations 52-54 gave the appropriate values of $a_{2}=6.6, a_{1}=4.75$, and $a_{0}=0.10$.

Substitution of the values of $D, V_{p}, k_{21}, k_{31}, \lambda_{1}, \lambda_{2}$, and $\lambda_{3}$ into equation 56 and simplification gave the equation 62 .

$$
\begin{aligned}
C_{p}^{\text {i.v. }}=21.1921225 e^{-0.021705074 t}+9.2944971 e^{-0.796904816 t} & +69.5633803 e^{-5.78139015 t}
\end{aligned}
$$


Substituting from equation 62 into equations 6,7 , and 12 gave equation 63.

$$
\begin{aligned}
V_{d \mathrm{ss}}= & 500,000\left[\frac{21.1921225}{(0.021705074)^{2}}+\frac{9.2444971}{(0.796904816)^{2}}+\frac{69.5633803}{(5.7813901)^{2}}\right] / \\
& {\left[\frac{21.1921225}{0.021705074}+\frac{9.2444971}{0.796909816}+\frac{69.5633803}{5.7813901}\right]^{2} } \\
= & 22,500
\end{aligned}
$$

For the particular three-compartment open model shown in Scheme II it is known that $V_{d \mathrm{ss}}$ is given by equation 64 .

$$
V_{d \mathrm{ss}}=\left(1+k_{12} / k_{21}+k_{13} / k_{31}\right) V_{p}=(1+3 / 2+1 / 0.5) 5000=22,500
$$

Hence the new equation yields the correct value of $V_{d s s}$ for the model shown in Scheme II. In general, for any of the three-compartment open models, where elimination may be from any compartment, $V_{d \mathrm{ss}}$ is given by equation 65 .

$$
V_{d \mathrm{ss}}=\frac{A_{b}^{\mathrm{ss}}}{C_{p}^{\overline{\mathrm{ss}}}}=\frac{\int_{0}^{\tau} A_{1}^{\mathrm{ss}} d t+\int_{0}^{\tau} A_{2}^{\mathrm{ss}} d t+\int_{0}^{\tau} A_{3}^{\mathrm{ss}} d t}{\int_{0}^{\tau} C_{p}^{\mathrm{ss}} d t}
$$

The value of $V_{d \mathrm{ss}}$ given by equation 12 will exactly coincide with the value given by equation 65 only when the particular three-compartment open model shown in Scheme II is involved. However, since we usually cannot determine from which compartments elimination occurs, this is part of the pharmacokineticist's dilemma. Hence unless there are good reasons for the contrary, the author suggests that $V_{d \mathrm{ss}}$ always be calculated by means of equation 12.

\section{DISCUSSION}

As discussed earlier $(1,5)$, there are many alternative models, all of which yield a two- or three-term exponential equation as illustrated by equations 3 and 4 . It is getting to be common practice to measure some pharmacological response as a function of time and occasionally to conclude that the response is related to the amount of drug in compartment 1 , compartment 2 , or compartment 3 . An alternative recommendation is to fit the data to the required number of exponential terms, then to determine if the response is approximately related to $A_{b}$ (from equation 17) or $A_{o}$ (from equations 17-19). This will tell one whether the site of action is in "the plasma compartment" or "some other part of the theoretical body" according to pharmacokinetic theory. However, the use of equations $17-19$ will in 
effect lump all peripheral compartments into one compartment. Whether this is a better alternative requires further study. It is important to know which compartment may relate to specific responses for specific drugs so that appropriate predictions and explanations may be made. To carry out such a determination accurately requires that plasma concentrations and responses be measured more than once shortly after intravenous administration as well as at later times. This is so since $A_{b}$ is a maximum at time of injection and falls off whenever $\boldsymbol{A}_{o}$ is zero at time zero, rises to a maximum at some later time, and then falls off. Hence the distinction is made readily with data collected shortly after intravenous administration.

Another point concerns degrees of freedom and what should be reported. If a bolus intravenous dose is administered and the plasma concentrations are fitted to a three-term exponential equation (equation 4), then one can report only six items as indicated by equation 4 , namely $C_{1}, C_{2}$, $C_{3}, \lambda_{1}, \lambda_{2}$, and $\lambda_{3}$.

Fitting the data to equation 4 is a model-independent process. However, one must realize that $\lambda_{i}$ values must be widely separated (i.e., greater than two- to fivefold) for one to accept the validity of any computer fit. A six-parameter equation probably requires 18 or more data points properly spaced throughout the $C_{p}, t$ curve. Assuming relatively accurate measurement, such data may yield a reasonable estimate of the parameters if the resultant $\lambda_{i}$ values are sufficiently separated as noted above. Otherwise, the computer-derived exponentials must be considered as only our tentative, probably inaccurate estimate of the appropriate equation.

If the data set fits the above restrictions, one can apply the equations listed in this article. Most of the equations 5-20 are model independent. However, equations 16 and 19 are model dependent, as noted earlier when they were originally discussed. Hence for consistency from one author to another it is here proposed that all authors use the general model shown in Scheme I and the general equations for this general model given in this article.

\section{Parameters}

$t_{1 / 2}$

The plasma half-life, $t_{1 / 2}$, may be defined as the time required for the plasma concentration to fall to one-half its value after absorption has ceased and pseudo-steady-state distribution has been attained. The latter, for practical purposes, means that the amount of drug in each peripheral compartment is falling off essentially at the same first-order rate as the amount of drug in the central (reference) compartment. Hence $t_{1 / 2}$ is the apparent elimination half-life. It is defined by equation 15 , and, in the 
symbolism section, $\lambda_{1}$ is defined as the smallest of the $\lambda_{i}$ values. If the "rules are obeyed" and terminal plasma concentrations are well fitted by the polyexponential equation, then this definition is satisfactory. It will be $\lambda_{1}$ which has the most influence of all the $\lambda_{i}$ values in determining $C_{\mathrm{ss}}^{\min }$ (see equation 37) and $\overline{C_{p}^{\text {ss }}}$ (see equations 39 and 10 ). The problem of not having a sensitive enough assay to "see" the correct value of $\lambda_{1}$, and the errors which ensue, has been discussed by Wagner (7). If $C_{p}, t$ data have been correctly computer-fitted to the general polynomial equation, this parameter, as with all other coefficients and exponentials, is really model independent, and does not rely on the general model shown in Scheme I.

Half-lives estimated from the other eigenvalues or $k_{i j}$ 's, such as $0.693 / \lambda_{2}$ and $0.693 / \lambda_{3}$, have frequently been termed "distribution halflives." But there are problems with such interpretations. If eigenvalues are involved, then they are a function of all the rate constants in the system and not just the microscopic distribution rate constants. If the coefficients and exponents of the polyexponential equation which is the "best fit" of each set of data are reported by an author, there appears to be no need to report the corresponding half-lives, since they may be readily calculated from the $\lambda_{i}$ values. The terminal $t_{1 / 2}$ (calculated from $\lambda_{1}$ ) is the lone exception, and the reason for this is that scientists are used to thinking in terms of an elimination half-life rather than in terms of an apparent first-order elimination rate constant.

$V_{d e x t}$

By the nature of its calculation (equation 14), $V_{d \text { ext }}$ is also model independent, and does not actually rely on the general model shown in Scheme I. However, one of the commonest errors made in the literature is to assume that after a bolus intravenous injection $\mathrm{Cl}_{p}=V_{d \text { ext }} \cdot \lambda_{1}$. This is true only when $V_{d \mathrm{ext}} \simeq V_{d \text { area }}$, as is the case with some drugs such as warfarin. The requirements for such a situation have been discussed by Albert et al. (8). When $V_{d \text { ext }}$ is appreciably larger than $V_{d \text { area }}$, then $V_{d \text { ext }}$ is of no use pharmacokinetically. When $V_{d \text { ext }} \simeq V_{d \text { area }}$, then $V_{d \text { ext }}$ is useful, since the latter parameter is more readily estimated (requiring fewer plasma assays) than $V_{d \text { area }}$.

$V_{p}$

If each set of data from a panel of subjects or patients is fitted with the appropriate number of exponential terms for each subject, then $V_{p}$ is also truly model independent, and does not rely on the general model shown in Scheme I. However, if one fits a given set of data to a biexponential equation when the data really require a triexponential equation, then the value of $V_{p}$ estimated from the two equations will be different. 
$V_{d \mathrm{ss}}$

The $V_{d s s}$ value calculated with equation 12 applies only to the general model shown in Scheme I. However, because of the dilemma of not knowing from which compartments there are exit rate constants to "outside the body," use of equation 12 will at least make all authors homogeneous in their approach.

$V_{d \text { area }}$

Equation 13 is based on mass balance considerations and is truly model independent when viewed in that light. However, $\left(A_{b}\right)_{\beta}=V_{d \text { area }} \cdot C_{p}$ in the terminal log-linear phase only for the general model shown in Scheme I.

$\mathrm{Cl}_{p}$

Equation 10 is based on mass balance considerations and is model independent when viewed in that light. However, $\mathrm{Cl}_{p}$ is equivalent to "total body clearance" only for the general model shown in Scheme I. If there were an exit rate constant, $k_{20}$, from compartment No. 2 of Scheme I, then total body clearance would be equal to $V_{1} k_{10}+V_{2} k_{20}$ and not equal to $\mathrm{Cl}_{p}$, as calculated with equation 10 . Many equate $\mathrm{Cl}_{p}$ with "total body clearance" without specifying under which conditions they are equivalent.

$A_{e}, A_{b}$, and $A_{o}$

Expressions $A_{e}, A_{b}$, and $A_{o}$ calculated with equations 16,17 , and 19 are dependent on the general model shown in Scheme I.

\section{Conclusion}

Most pharmacokinetic articles on specific drugs report some or all of the parameters discussed above. The advantage of the equations in this article is that all of them may be calculated directly from the coefficients and exponents of the polyexponential equation without deriving any microscopic rate constants.

\section{APPENDIX}

Derivation for $\boldsymbol{A}_{\boldsymbol{b}}$ After Bolus Intravenous Dose

$$
A_{b}=D_{\text {i.v. }}-\mathrm{Cl}_{p} \int_{0}^{t} C_{p} d t
$$


Now,

$$
\int_{0}^{t} C_{p} d t=\int_{0}^{t} \sum C_{i} e^{-\lambda_{i} t} d t^{\prime}=\sum \frac{C_{i}}{\lambda_{i}}\left(1-e^{-\lambda_{i} t}\right)
$$

Substituting from equation 10 for $\mathrm{Cl}_{p}$ and for the integral from equation 67 into equation 66 gives

$$
\begin{aligned}
A_{b} & =D_{\text {i.v. }}-\left[D_{\text {i.v. }} \sum \frac{C_{i}}{\lambda_{i}}\left(1-e^{-\lambda_{i} t}\right) / \sum C_{i} / \lambda_{i}\right] \\
& =D_{\text {i.v. }}\left[1-\sum \frac{C_{i}}{\lambda_{i}}\left(1-e^{-\lambda_{i} t}\right) / \sum C_{i} / \lambda_{i}\right] \\
& =D_{\text {i.v. }}\left[\sum C_{i} / \lambda_{i}-\sum \frac{C_{i}}{\lambda_{i}}+\sum \frac{C_{i}}{\lambda_{i}} e^{-\lambda_{i} t} / \sum C_{i} / \lambda_{i}\right] \\
& =D_{\text {i.v. }} \sum \frac{C_{i}}{\lambda_{i}} e^{-\lambda_{i} t} / \sum C_{i} / \lambda_{i}=D_{\text {i.v. }} \cdot S_{4} / S_{2}
\end{aligned}
$$

\section{Derivation for $\boldsymbol{V}_{d s s}$ After Bolus Intravenous Injection}

$$
\mathrm{A}_{b}^{\overline{\mathrm{ss}}}=\int_{0}^{\tau} A_{b}^{\mathrm{ss}} d t / \tau=D \sum \int_{0}^{\tau} \frac{C_{i}}{\lambda_{i}\left(1-e^{-\lambda_{i} \tau}\right)} e^{-\lambda_{i} t} d t / \tau \sum C_{i} / \lambda_{i}
$$

Omitting the sum sign temporarily, we find that

$$
\begin{aligned}
\int_{0}^{\tau} \frac{C_{i}}{\lambda_{i}\left(1-e^{-\lambda_{i} \tau}\right)} e^{-\lambda_{i} t} d t & =\left|-\frac{C_{i}}{\lambda_{i}^{2}\left(1-e^{-\lambda_{i} \tau}\right)} e^{-\lambda_{i} t}\right|_{0}^{\tau} \\
& =-\frac{C_{i}}{\lambda_{i}^{2}\left(1-e^{-\lambda_{i} \tau}\right)} e^{-\lambda_{i} \tau}-\left[-\frac{C_{i}}{\lambda_{i}^{2}\left(1-e^{-\lambda_{i} \tau}\right)}\right] \\
& =\frac{C_{i}}{\lambda_{i}^{2}\left(1-e^{-\lambda_{i} \tau}\right)}\left[1-e^{-\lambda_{i} \tau}\right]=C_{i} / \lambda_{i}^{2}
\end{aligned}
$$

Hence

$$
A_{b}^{\overline{\mathrm{ss}}}=\left[\frac{D_{\mathrm{i} . \mathrm{v}}}{\tau} \sum C_{i} / \lambda_{i}^{2}\right] / \sum C_{i} / \lambda_{i}
$$

Also,

$$
\begin{gathered}
C_{p}^{\overline{\mathrm{ss}}}=\frac{\int_{0}^{\tau} C_{p}^{\mathrm{ss}} d t}{\tau}=\frac{\int_{0}^{\infty} C_{p} d t}{\tau}=\frac{\sum C_{i} / \lambda_{i}}{\tau} \\
V_{d \mathrm{ss}}=\frac{\mathrm{A}_{b}^{\overline{\mathrm{ss}}}}{C_{p}^{\overline{\mathrm{ss}}}}=\frac{\left[\frac{D_{\text {i.v. }}}{\tau} \sum C_{i} / \lambda_{i}^{2}\right] / \sum C_{i} / \lambda_{i}}{\left[\sum C_{i} / \lambda_{i}\right] / \tau}=\frac{D_{\text {i.v. }} \sum C_{i} / \lambda_{i}^{2}}{\left[\sum C_{i} / \lambda_{i}\right]^{2}}=D_{\text {i.v. }} \cdot S_{3} /\left(S_{2}\right)^{2}
\end{gathered}
$$




\section{Time-Dependent Volume of Distribution After Bolus Intravenous Dose (2)}

$\left(V_{d}\right) t$ is obtained by making a ratio of the above expressions for $A_{b}$ and $C_{p}$ with the result below.

$\left(V_{d}\right) t=\frac{A_{b}}{C_{p}}=D_{\text {i.v. }} \sum \frac{C_{i}}{\lambda_{i}} e^{-\lambda_{i} t} /\left[\sum C_{i} e^{-\lambda_{i} t}\right]\left[\sum C_{i} / \lambda_{i}\right]=D_{\text {i,v. }} \cdot S_{4} / C_{p}^{\text {i.v. }} \cdot S_{2}$

In the $\log$-linear phase only $\lambda_{1}$ remains and $\left(V_{d}\right)_{t \beta}$ is given by equation 11.

Example Showing Relationship Between Coefficients for $\boldsymbol{C}_{p y} \boldsymbol{t}$ Equations During Constant-Rate Infusion and After Bolus Intravenous Injection

Take as example the two-compartment open model.

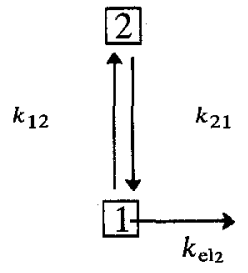

Scheme III. Bolus intravenous injection.

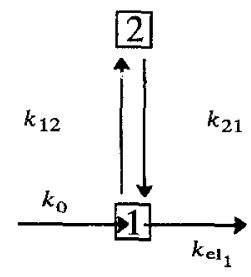

Scheme IV. During infusion.

After bolus intravenous injection, equation 40 applies, where

$$
\begin{gathered}
\lambda_{1}+\lambda_{2}=k_{12}+k_{21}+k_{\mathrm{el}} \\
\lambda_{1} \lambda_{2}=k_{21} k_{\mathrm{el}}
\end{gathered}
$$

If we write equation 40 as equation 77 ,

$$
C_{p}=C_{1} e^{-\lambda_{1} t}+C_{2} e^{-\lambda_{2} t}
$$

then by matching coefficients, we find

$$
\begin{gathered}
C_{1}=\frac{D\left(k_{21}-\lambda_{1}\right)}{V_{p}\left(\lambda_{2}-\lambda_{1}\right)} \\
C_{2}=\frac{-D\left(k_{21}-\alpha\right)}{V_{p}\left(\lambda_{2}-\lambda_{1}\right)}
\end{gathered}
$$


During a constant-rate intravenous infusion, equation 80 applies:

$$
\begin{aligned}
C_{p} & =\frac{k_{0}\left(k_{21}-\lambda_{1}\right)\left(1-e^{+\lambda_{1} t}\right)}{-\lambda_{1}\left(\lambda_{2}-\lambda_{1}\right) V_{p}} e^{-\lambda_{1} t}+\frac{k_{0}\left(k_{21}-\lambda_{2}\right)\left(1-e^{+\lambda_{2} t}\right)}{-\lambda_{2}\left(\lambda_{1}-\lambda_{2}\right) V_{p}} e^{-\lambda_{2} t} \\
& =\frac{k_{0}\left(k_{21}-\lambda_{1}\right)}{\lambda_{1}\left(\lambda_{2}-\lambda_{1}\right) V_{p}}\left(1-e^{-\lambda_{1} t}\right)+\frac{k_{0}\left(k_{21}-\lambda_{2}\right)}{\lambda_{2}\left(\lambda_{1}-\lambda_{2}\right) V_{p}}\left(1-e^{-\lambda_{2} t}\right)
\end{aligned}
$$

We may write equation 80 as equation 81 .

$$
C_{p}=X_{1}\left(1-e^{-\lambda_{1} t}\right)+X_{2}\left(1-e^{-\lambda_{2} t}\right)
$$

By matching coefficients of equations 80 and 81 , we find

$$
\begin{gathered}
X_{1}=\frac{k_{0}\left(k_{21}-\lambda_{1}\right)}{\lambda_{1}\left(\lambda_{2}-\lambda_{1}\right) V_{p}} \\
X_{2}=\frac{k_{0}\left(k_{21}-\lambda_{2}\right)}{\lambda_{2}\left(\lambda_{1}-\lambda_{2}\right) V_{p}}=-\frac{k_{0}\left(k_{21}-\lambda_{1}\right)}{\lambda_{2}\left(\lambda_{2}-\lambda_{1}\right) V_{p}}
\end{gathered}
$$

From the bolus intravenous equation 40 for the same model, we can see that

$$
\begin{gathered}
C_{1}=\frac{D_{\text {i.v. }}\left(k_{21}-\lambda_{1}\right)}{\left(\lambda_{2}-\lambda_{1}\right) V_{p}} \\
C_{2}=\frac{-D_{\text {i.v. }}\left(k_{21}-\lambda_{1}\right)}{\left(\lambda_{2}-\lambda_{1}\right) V_{p}}
\end{gathered}
$$

Also,

$$
k_{0}=\frac{D_{\text {i.v. }}}{T}
$$

Utilizing equations 82,84 , and 86 , we obtain equations $87-89$.

$$
\begin{gathered}
\frac{X_{1}}{C_{1}}=\frac{D_{\mathrm{i}, \mathrm{v} .}\left(k_{21}-\lambda_{1}\right)}{\lambda_{1} T\left(\lambda_{2}-\lambda_{1}\right) V_{p}} \cdot \frac{\left(\lambda_{2}-\lambda_{1}\right) V_{p}}{D_{\mathrm{i}, \mathrm{v} .}\left(k_{21}-\lambda_{1}\right)}=\frac{1}{\lambda_{1} T} \\
X_{1}=C_{1} / \lambda_{1} T \\
C_{1}=\lambda_{1} T X_{1}
\end{gathered}
$$

Utilizing equations 83,85 , and 86 , we obtain equations 90-92.

$$
\begin{gathered}
\frac{X_{2}}{C_{2}}=\left[\frac{-D_{\text {i.v. }}\left(k_{21}-\lambda_{1}\right)}{\lambda_{2} T\left(\lambda_{2}-\lambda_{1}\right) V_{p}}\right]\left[\frac{-\left(\lambda_{2}-\lambda_{1}\right) V_{p}}{D_{\text {i.v. }}\left(k_{21}-\lambda_{1}\right)}\right]=\frac{1}{\lambda_{2} T} \\
X_{2}=C_{2} / \lambda_{2} T \\
C_{2}=\lambda_{2} T X_{2}
\end{gathered}
$$


Hence we may write the infusion equations as shown in Table I and equation 21.

Post-intravenous-infusion equation 93 applies to Scheme IV.

$$
\begin{aligned}
C_{p} & =\frac{k_{0}\left(k_{21}-\lambda_{1}\right)\left(1-e^{+\lambda_{1} T}\right)}{-\lambda_{1}\left(\lambda_{2}-\lambda_{1}\right) V_{p}} e^{-\lambda_{1} t}+\frac{k_{0}\left(k_{21}-\lambda_{2}\right)\left(1-e^{+\lambda_{2} T}\right)}{-\lambda_{2}\left(\lambda_{1}-\lambda_{2}\right) V_{p}} e^{-\lambda_{2} t} \\
& =\frac{k_{0}\left(k_{21}-\lambda_{1}\right)\left(e^{+\lambda_{1} T}-1\right)}{\lambda_{1}\left(\lambda_{2}-\lambda_{1}\right) V_{p}} e^{-\lambda_{1} t}+\frac{k_{0}\left(k_{21}-\lambda_{2}\right)\left(e^{+\lambda_{2} T}-1\right)}{\lambda_{2}\left(\lambda_{1}-\lambda_{2}\right) V_{p}} e^{-\lambda_{2} t}
\end{aligned}
$$

We may write equation 93 as equation 94 .

$$
C_{p}=Y_{1} e^{-\lambda_{1} t}+Y_{2} e^{-\lambda_{2} t}
$$

By matching coefficients in equations 93 and 94 , we find

$$
\begin{gathered}
Y_{1}=\frac{k_{0}\left(k_{21}-\lambda_{1}\right)\left(e^{+\lambda_{1} T}-1\right)}{\lambda_{1}\left(\lambda_{2}-\lambda_{1}\right) V_{p}} \\
Y_{2}=\frac{k_{0}\left(k_{21}-\lambda_{2}\right)\left(e^{+\lambda_{2} T}-1\right)}{\lambda_{2}\left(\lambda_{1}-\lambda_{2}\right) V_{p}}=-\frac{k_{0}\left(k_{21}-\lambda_{2}\right)\left(e^{+\lambda_{2} t}-1\right)}{\lambda_{2}\left(\lambda_{2}-\lambda_{1}\right) V_{p}}
\end{gathered}
$$

Utilizing equations 84,86 , and 95 , we obtain equations $97-99$.

$$
\begin{gathered}
\frac{Y_{1}}{C_{1}}=\frac{D_{\text {i.v. }}\left(k_{21}-\lambda_{1}\right)\left(e^{+\lambda_{1} T}-1\right)}{\lambda_{1} T\left(\lambda_{2}-\lambda_{1}\right) V_{p}} \cdot \frac{\left(\lambda_{2}-\lambda_{1}\right) V_{p}}{D_{\text {i.v. }}\left(k_{21}-\lambda_{1}\right)}=\frac{e^{+\lambda_{1} T}-1}{\lambda_{1} T} \\
Y_{1}=C_{1}\left\{\frac{e^{+\lambda_{\mathrm{r}} T}-1}{\lambda_{1} T}\right\} \\
C_{1}=\frac{\lambda_{1} T Y_{1}}{e^{+\lambda_{1} T}-1}
\end{gathered}
$$

Utilizing equations 83,86 , and 96 , we obtain equations $100-102$.

$$
\begin{gathered}
\frac{Y_{2}}{C_{2}}=\left[\frac{-D_{\text {i.v. }}\left(k_{21}-\lambda_{2}\right)\left(e^{+\lambda_{2} T}-1\right)}{\lambda_{2} T\left(\lambda_{2}-\lambda_{1}\right) V_{p}}\right]\left[\frac{-\left(\lambda_{2}-\lambda_{1}\right) V_{p}}{D_{\text {i.v. }}\left(k_{21}-\lambda_{2}\right)}\right]=\frac{e^{+\lambda_{2} T}-1}{\lambda_{2} T} \\
Y_{2}=C_{2}\left\{\frac{e^{+\lambda_{2} T}-1}{\lambda_{2} T}\right\} \\
C_{2}=\frac{\lambda_{2} T Y_{2}}{e^{+\lambda_{2} T}-1}
\end{gathered}
$$

Hence we may write the infusion equations as shown in Table I and equation 22. 


\section{REFERENCES}

1. J. G. Wagner. Linear pharmacokinetic models and vanishing exponential terms: Implications in pharmacokinetics. J. Pharmacokin. Biopharm. 4:395-425 (1976).

2. S. Niazi. Volume of distribution as a function of time. J. Pharm. Sci. 65:452-454 (1976).

3. A. J. Sedman and J. G. Wagner. CSTRIP, a fortran IV computer program for obtaining initial polyexponential parameter estimates. J. Pharm. Sci. 65:1006-1010 (1976).

4. J. G. Wagner. A safe method for rapidly achieving plasma concentration plateaus. Clin. Pharmacol. Ther. 16:691-700 (1974).

5. J. G. Wagner. Do you need a pharmacokinetic model, and if so, which one? J. Pharmacokin. Biopharm. 3:457-478 (1975)

6. J. G. Wagner, J. I. Northam, C. D. Alway, and O. S. Carpenter. Blood levels of drug at the equilibrium state after multiple dosing. Nature 207:1301-1302 (1965).

7. J. G. Wagner. Reply to letter concerning the definition of half-life of a drug. Drug. Intell. Clin. Pharm. 7:357-358 (1973).

8. K. S. Albert, A. J. Sedman, and J. G. Wagner. Pharmacokinetics of orally administered acetaminophen in man. J. Pharmacokin. Biopharm. 2:381-393 (1974). 